\title{
Spreading of correlations and entanglement in the long-range transverse Ising chain
}

\author{
J. T. Schneider $\odot,,^{1, *}$ J. Despres, ${ }^{1}$ S. J. Thomson $\odot,{ }^{1}$ L. Tagliacozzo $\odot,{ }^{2,3}$ and L. Sanchez-Palencia $\oplus^{1}$ \\ ${ }^{1}$ CPHT, CNRS, Ecole Polytechnique, IP Paris, F-91128 Palaiseau, France \\ ${ }^{2}$ Department of Physics and SUPA, University of Strathclyde, Glasgow G4 ONG, United Kingdom \\ ${ }^{3}$ Departament de Física Quàntica i Astrofísica and Institut de Ciències del Cosmos (ICCUB), \\ Universitat de Barcelona, Martí i Franquès 1, E-08028 Barcelona, Catalonia, Spain
}

(Received 23 November 2020; accepted 28 January 2021; published 5 March 2021)

\begin{abstract}
Whether long-range interactions allow for a form of causality in nonrelativistic quantum models remains an open question with far-reaching implications for the propagation of information and thermalization processes. Here, we study the out-of-equilibrium dynamics of the one-dimensional transverse Ising model with algebraic long-range exchange coupling. Using a state of the art tensor-network approach, complemented by analytic calculations and considering various observables, we show that a weak form of causality emerges, characterized by nonuniversal dynamical exponents. While the local spin and spin correlation causal edges are sub-ballistic, the causal region has a rich internal structure, which, depending on the observable, displays ballistic or superballistic features. In contrast, the causal region of entanglement entropy is featureless and its edge is always ballistic, irrespective of the interaction range. Our results shed light on the propagation of information in long-range interacting lattice models and pave the way to future experiments, which are discussed.
\end{abstract}

DOI: 10.1103/PhysRevResearch.3.L012022

Long-range interactions may dramatically impact the dynamics of correlated systems [1]. In the quantum regime, a number of basic concepts, such as the equivalence of the thermodynamic ensembles [2], the Mermin-Wagner-Hohenberg theorem [3-5], or the area law for entanglement entropy [6-9], break down. Long-range interactions may also be responsible for negative heat capacities and anomalous response functions [10]. The paradigmatic model considers interactions that fall off algebraically with the distance $R$, as $1 / R^{\alpha}$. Such long-range interactions can now be emulated in quantum simulators [11-15] with artificial ion crystals [13,1620], Rydberg gases [21-24], magnetic atoms [25-29], polar molecules [30-32], nonlinear optical media [33], and solidstate defects [34-36]. A major asset of these systems is that they are free of screening effects and the interaction is truly long range. Moreover, the exponent $\alpha$ can be controlled [16,24].

Another dramatic effect of long-range interactions is the breakdown of the notion of causality in nonrelativistic quantum models. For a wide class of lattice models with short-range interactions, the Lieb-Robinson bound implies that correlations decay exponentially beyond a limit set by the system's maximum group velocity [37-39]. This effective light-cone effect has been demonstrated for various models in both experiments [40-42] and numerics [43-49].

\footnotetext{
${ }^{*}$ Corresponding author: jan.schneider@polytechnique.edu

Published by the American Physical Society under the terms of the Creative Commons Attribution 4.0 International license. Further distribution of this work must maintain attribution to the author(s) and the published article's title, journal citation, and DOI.
}

In contrast, for sufficiently long-range interactions, causality breaks down and information can propagate arbitrarily fast $[50,51]$. This is consistent with the absence of known Lieb-Robinson bounds [38,52,53] and the vanishing of the characteristic dynamical time scale in the thermodynamic limit [54]. Conversely, algebraic interactions that decay fast enough are effectively short range, and one recovers ballistic propagation of information [55-57]. The intermediate regime is, however, strongly debated, and whether a form of causality emerges remains an open question. Known bounds in principle allow for super-ballistic propagation $[38,52,53]$ but they are challenged by numerical simulations, which point towards a significantly slower propagation $[9,50,58-62]$. The latter, however, reported different propagation scaling laws. Microscopic mean-field theory suggests that these apparent contradictions may be attributed to the coexistence of several signals governed by different dynamical scaling laws [63]. This prediction, however, relies on a generic but nonuniversal form of the correlation functions and ignores beyond-meanfield effects. Experiments performed with trapped ions have reported bounded propagation $[64,65]$ but they are limited to very small systems, which prevents extraction of the scaling laws and closure of the debate.

The aim of this work is to characterize the spreading of quantum correlations in the intermediate regime of a longrange spin system numerically. Specifically, we determine the scaling laws for the propagation of a variety of observables in the long-range transverse Ising (LRTI) chain using matrix product state simulations. We find that a weak form of causality emerges, characterized by generic algebraic scaling forms $t \sim R^{\beta}$, where the specific value of the exponent $\beta$ depends on the observable. The spin-correlation and local-spin causal edges are both sub-ballistic, with the same dynamical 


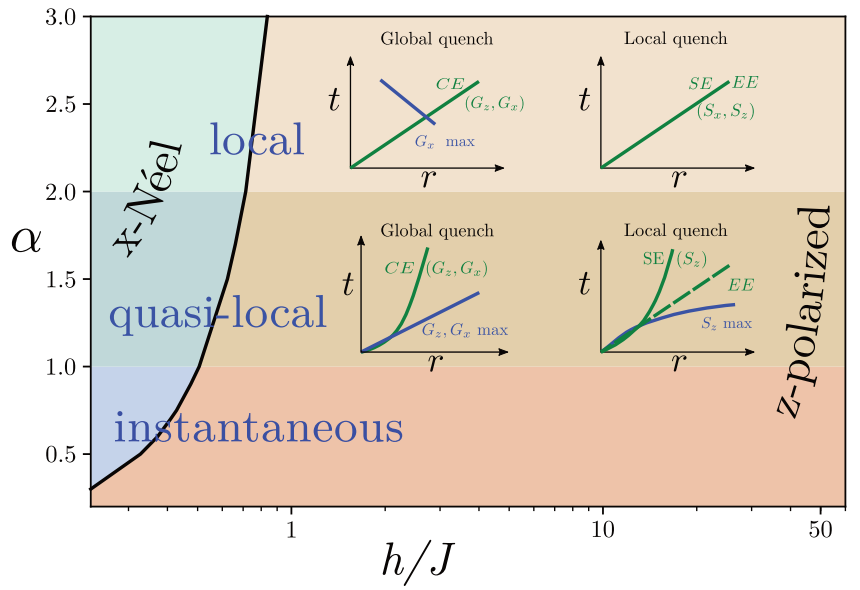

FIG. 1. Phase diagram and dynamical properties of the LRTI chain in the $(h / J, \alpha)$ plane. The $x$-Néel phase (shades of blue) is separated from the $z$-polarized phase (shades of orange) by a critical line in black. The three dynamical regimes are shaded differently. As shown in the insets, the local and quasilocal regimes are characterized by distinct algebraic scaling laws for the correlation and spin edges (CE and SE, respectively), their maxima in space-time ( $\max$ ), and the entanglement edge (EE), after global or local quench. The different behaviors of these observables in different regions of the phase diagram provide clear, experimentally accessible, signatures of the local and quasilocal regimes.

exponent $\left(\beta_{\mathrm{CE}}=\beta_{\mathrm{SE}}>1\right)$. In the vicinity of the edge, however, the local maxima propagate differently, i.e., ballistically $\left(\beta_{\mathrm{m}}=1\right)$ for spin-spin correlations and super-ballistically $\left(\beta_{\mathrm{m}}<1\right)$ for local spins. In contrast, the Rényi entanglement entropies always propagate ballistically, irrespective of the range of interactions, in both the local and quasilocal regime. The analytic quasiparticle picture, based on linear spin wave theory (LSWT), accurately reproduces the numerics and provides a clear interpretation of the numerical results. The different algebraic space-time patterns of correlation functions provide an unambiguous fingerprint of the dynamical regimes of the model, suggesting the emergence of a dynamical phase diagram. These correlation patterns can be directly measured in state-of-the-art experiments.

Model and approach. The dynamics of the LRTI chain is governed by the Hamiltonian,

$$
\hat{H}=\sum_{R \neq R^{\prime}} \frac{J}{\left|R-R^{\prime}\right|^{\alpha}} \hat{S}_{R}^{x} \hat{S}_{R^{\prime}}^{x}-2 h \sum_{R} \hat{S}_{R}^{z},
$$

where $\hat{S}_{R}^{j}(j=x, y, z)$ are the spin-1/2 operators on lattice site $R \in[0, N-1], N$ is the system size, $J>0$ is the coupling energy, and $h$ is the transverse field. It can be realized on various quantum simulation platforms, including cold Rydberg gases [24,66,67] and artificial ion crystals, where the exponent $\alpha$ can be controlled via light-mediated interactions $[16,18,19,64,65]$. At equilibrium, the phase diagram of the LRTI chain comprises two gapped phases separated by a second-order quantum phase transition; see Fig. 1 and Ref. [7]. For low fields $h$ and rather short-range couplings (high values of $\alpha$ ), the nearest-neighbor antiferromagnetic couplings dominate and the system forms a staggered Néel-
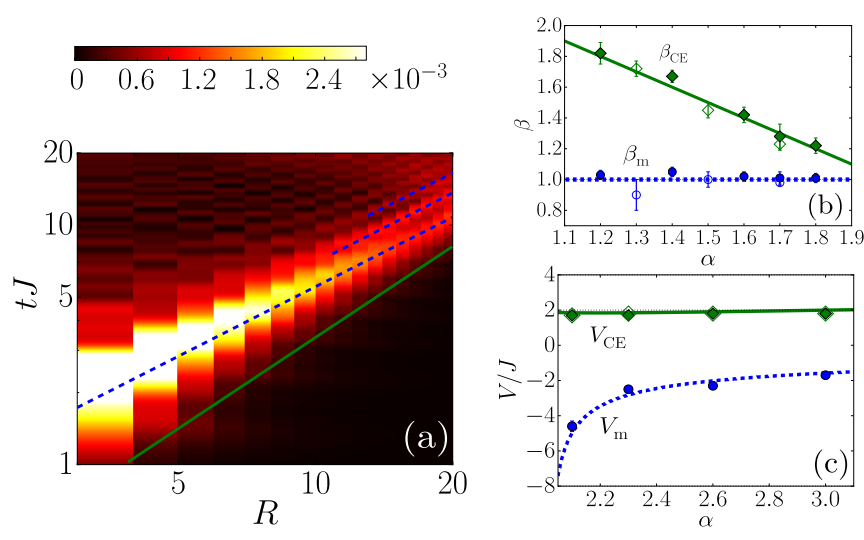

FIG. 2. Spreading of spin correlations in a global quench, with system size $N=48$. (a) TDVP results for $G_{z}$ and a quench from $(h / J)_{\mathrm{i}}=50$ to $(h / J)_{\mathrm{f}}=1$ in the quasilocal regime at $\alpha=1.7$. The solid green and dashed blue lines are fits to the $\mathrm{CE}$ and the extrema, respectively. (b) Dynamical exponents $\beta_{\mathrm{CE}}$ (green diamonds) and $\beta_{\mathrm{m}}$ (blue disks), fitted form results as in panel (a) for $G_{z}$ (empty symbol) and $G_{x}$ (full symbol), and comparison to the LSWT predictions (solid green and dashed blue lines). (c) Spreading velocities $V_{\mathrm{CE}}$ (green diamonds) and $V_{\mathrm{m}}$ (blue disks), in the local regime and comparison to the LSWT predictions (solid green and dashed blue lines).

ordered phase along the $x$ direction. For a large field $h$ and long-range couplings (low values of $\alpha$ ), the spin-field interaction is favored and a $z$-polarized phase is formed.

Out of equilibrium, the LSWT predicts three dynamical regimes (shaded by different colors in fig. 1) [50,58]: For $\alpha \geqslant 2$ (local regime), the spin wave excitations are regular with bounded energies $E_{k}$ and group velocities $V_{\mathrm{g}}(k)=\partial_{k} E_{k}$ ( $k$ is the momentum). This regime is reminiscent of the shortrange case where correlations spread at finite speed, giving rise to a linear causality cone [37,38]. For $\alpha<1$ (instantaneous regime), $E_{k}$ features an algebraic, infrared divergence. There is no characteristic time scale and correlations spread arbitrarily fast. Finally, for $1 \leqslant \alpha<2$ (quasilocal regime), $E_{k}$ is bounded but $V_{\mathrm{g}}(k)$ diverges as $V_{\mathrm{g}}(k) \sim 1 / k^{2-\alpha}$. Whether some form of causality emerges in the quasilocal regime remains debated [50,54,58,60,64,65,68], and, in the following, we mainly focus on this case.

Except where otherwise indicated, the results discussed below are obtained using time-dependent variational principle (TDVP) simulations within a matrix-product state (MPS) framework $[7,50,69]$. Convergence of the calculations with the bond dimension has been systematically checked. Our results are summarized on the dynamical phase diagram of Fig. 1. The main features of the observable that we consider are all described by an algebraic space-time dependence of their edges and maxima, shown in the insets.

Spin correlations and global quench. We first consider the spreading of the connected spin correlations, $\quad G_{j}(R, t)=G_{j}^{0}(R, t)-G_{j}^{0}(R, 0), \quad$ with $G_{j}^{0}(R, t)=$ $\left\langle\hat{S}_{R}^{j}(t) \hat{S}_{0}^{j}(t)\right\rangle-\left\langle\hat{S}_{R}^{j}(t)\right\rangle\left\langle\hat{S}_{0}^{j}(t)\right\rangle$, along the directions $j=x, z$. Figure 2(a) shows a typical TDVP result for $G_{z}(R, t)$, for a global quench in the quasilocal regime, $\alpha=1.7$, from $(h / J)_{\mathrm{i}}=50$ to $(h / J)_{\mathrm{f}}=1$, both in the $z$-polarized phase. The initial state of the system is the ground state of the 
Hamiltonian with $(h / J)_{\mathrm{i}}$. Similar results are found when scanning the values of $(h / J)_{\mathrm{i}},(h / J)_{\mathrm{f}}$, and $1<\alpha<2$. The correlation pattern shows a series of maxima which propagate algebraically [notice the log-log scale in Fig. 2(a)]. In the asymptotic long-time and long-distance limits, they are well fitted by the scaling law $t \sim R^{\beta_{\mathrm{m}}}$ (dashed blue lines). The correlation edge (CE), which sets the causality horizon, cannot, however, be inferred from the behavior of the maxima [47]. To find the CE, we track the points of the $R-t$ plane where the correlations reach a fraction $\epsilon$ of their maximum. Scanning $\epsilon \in[0.01,0.1]$, we find that the $\mathrm{CE}$ is well fitted by the algebraic scaling law $t \sim R^{\beta_{\mathrm{CE}}}$ (solid line), with $\beta_{\mathrm{CE}}$ nearly independent of $\epsilon$ [70]. Figure 2(b) shows the results of the fits (empty symbols) versus the exponent $\alpha$.

The numerical results for $G_{x}(R, t)$ have an extra checkerboard structure inside the causal region due to the antiferromagnetic coupling in Eq. (1). Once this structure is taken into account, we can identify a CE and local maxima, and extract the corresponding exponents. The results are similar to those found for $G_{z}(R, t)$; see filled symbols in Fig. 2(b) [70].

Comparing the fitted dynamical exponents $\beta_{\mathrm{m}}$ and $\beta_{\mathrm{CE}}$ to the predictions of the LSWT [63], we find an excellent agreement, as shown in Fig. 2(b). While the maxima spread ballistically, $\beta_{\mathrm{m}} \simeq 1$, the $\mathrm{CE}$ is sub-ballistic with $\beta_{\mathrm{CE}} \simeq$ $\beta_{\text {LSWT }}=3-\alpha>1$. This is characteristic of gapped longrange models, such as the LRTI model in the $z$-polarized phase [63]. It confirms the emergence of a weak, slower-thanballistic form of causality for $1<\alpha<2$.

In the local regime, $\alpha \geqslant 2$, we find that both the maxima and the $\mathrm{CE}$ spread ballistically, $\beta_{\mathrm{m}} \simeq \beta_{\mathrm{CE}} \simeq 1$. The spreading velocities are, however, different from each other, which is characteristic of a nonphononic excitation spectrum. The $\mathrm{CE}$ velocity is $V_{\mathrm{CE}} \simeq 2 V_{\mathrm{g}}\left(k^{*}\right)$, i.e., twice the maximum group velocity of the spin waves, while that of the maxima is $V_{\mathrm{m}} \simeq$ $2 V_{\varphi}\left(k^{*}\right)$, i.e., twice the phase velocity $V_{\varphi}(k)=E_{k} / k$ at the quasimomentum $k^{*}$ where the group velocity is maximum; see Fig. 2(c).

We have also performed calculations for large quenches. While the LSWT is well justified only for weak quenches, we have found that the dynamical exponents it predicts are extremely robust, even for quenches across the critical line. We found dynamical scaling laws in very good agreement with those reported on Figs. 2(b) and 2(c), although the signal is blurred as compared to weak quenches owing to the proliferation of quasiparticles when the quench amplitude increases [70].

Local magnetization and local quench. We now consider the dynamics of another quantity, namely the local magnetization $\left\langle\hat{S}_{R}^{z}(t)\right\rangle$, and perform a local quench. We initialize the system in the ground state of the $z$-polarized phase and flip the central spin. Figure 3(a) shows a typical TDVP result for the quantity $1 / 2-\left\langle\hat{S}_{R}^{z}(t)\right\rangle$ versus the time $t$ and the distance $R$ from the flipped spin, in the quasilocal regime. The result displays, as in the case of global quenches, a twofold algebraic structure. Fitting the maxima (dashed blue line) and the spin edge ( $\mathrm{SE}$, solid green line) as previously, we extract the dynamical exponents $\beta_{\mathrm{m}}$ and $\beta_{\mathrm{SE}}$ plotted in Fig. 3(b) (blue disks and green diamonds, respectively). The SE follows the same sub-ballistic scaling law as the CE in the previous case, $\beta_{\mathrm{SE}} \simeq 3-\alpha$ (solid green line). In contrast, the maxima of the
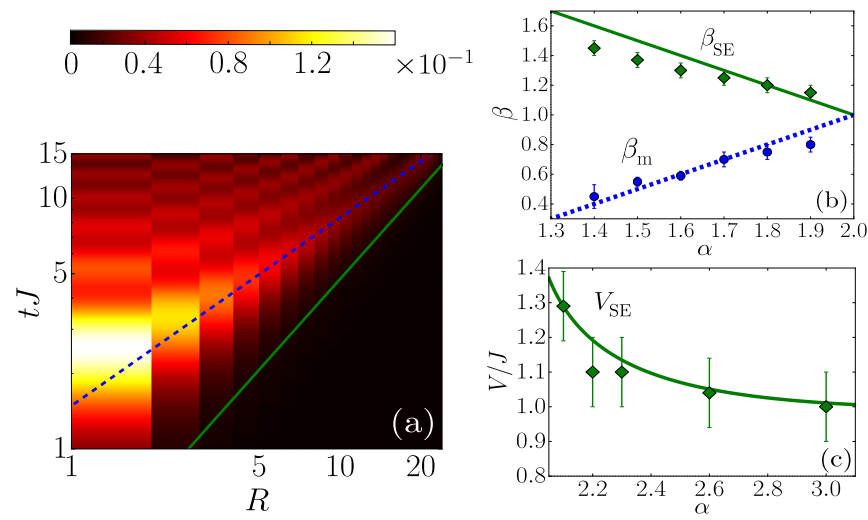

FIG. 3. Spreading of the local magnetization for a local quench, with system size $N=48$. (a) TDVP results for the quantity $1 / 2-$ $\left\langle\hat{S}_{R}^{z}(t)\right\rangle$ versus the time and the distance from the flipped spin for $h / J=50$ and $\alpha=1.8$. (b) Dynamical exponents $\beta_{\mathrm{SE}}$ (green diamonds) and $\beta_{\mathrm{m}}$ (blue disks), fitted form results as in (a) and comparison to the LSWT predictions (solid green and dashed blue lines). (c) Spreading velocity $V_{\mathrm{SE}}$ (green diamonds) and comparison to the LSWT prediction in the local regime (solid green line).

local spin spreads faster than those of the spin correlations, and here we find $\beta_{\mathrm{m}}<1$, corresponding to a super-ballistic propagation.

To understand this behavior, let us first recall that the standard LSWT generically predicts a super-ballistic propagation of the maxima in gapless systems [63]. Here, although the LRTI model is gapped, the initial state $\left|\Psi_{0}\right\rangle \simeq$ $|\uparrow \cdots \uparrow \downarrow \uparrow \cdots \uparrow\rangle$ of the local quench is orthogonal to the ground state $\left|\Psi_{\mathrm{GS}}\right\rangle \simeq|\uparrow \uparrow \cdots \uparrow\rangle$, and thus lives in the first excited manifold. The ground state and the gap are thus irrelevant, and we may expect super-ballistic propagation of the maxima consistent with the TDVP results. This argument applies to any observable for such a local quench. More specifically, the LSWT applied to $\left\langle\hat{S}_{R}^{z}(t)\right\rangle$ for the local quench yields $\beta_{\mathrm{m}}=\alpha-1$, in very good agreement with the TDVP results; see Fig. 3(b) [70].

In the local regime $(\alpha>2)$ we find that $\left\langle\hat{S}_{\tilde{R}}^{z}(t)\right\rangle$ propagates ballistically. Both this property and the SE velocity extracted from the TDVP calculations are in good agreement with the LSWT analysis; see Fig. 3(c). Note that in this regime, we do not observe maxima propagating at a different velocity. This is also consistent with the LSWT analysis. In the local regime, the quantity $\left\langle\hat{S}_{R}^{z}(t)\right\rangle$ is the sum of several contributions, each with a twofold structure but that are in phase quadrature and cancel each other. A similar effect has been found for density correlations deep in the Mott insulator phase of the BoseHubbard model [47,70].

Entanglement entropy. We finally study the spreading of quantum information after the same local quench. It may be measured via the Rényi entropies of the reduced density matrix of a block,

$$
\mathcal{S}_{n}(R, t)=\frac{1}{1-n} \log \left\{\operatorname{tr}\left[\hat{\rho}^{n}(R, t)\right]\right\},
$$

with $n>0$ and $\hat{\rho}(R, t)=\hat{\rho}_{A}=\operatorname{tr}_{B}(|\Psi(t)\rangle\langle\Psi(t)|)$ the reduced density matrix at time $t$ of the subsystem $A=[R, R+$ $1, \ldots, N / 2]$. The position $R$ is measured from the flipped 

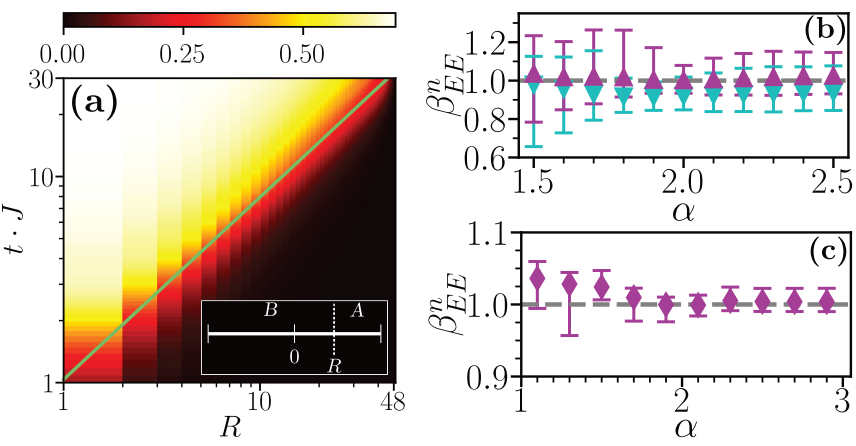

FIG. 4. Spreading of the von Neumann entanglement entropy $\mathcal{S}_{n=1}(R, t)$ for the same quench as in Fig. 3(a). (a) TDVP results for $h / J=50, \alpha=1.8$, and a system size $N=96$. The solid green line marks a power law fit to the EE with $\epsilon=0.5$, yielding $\beta_{\mathrm{EE}}^{n}=$ $0.899 \pm 0.005$. (b) Dynamical exponents for the EE obtain via TDVP (cyan downwards triangles) and LSWT (magenta upwards triangles), with error bars corresponding to the variations with respect to the threshold $\epsilon$. (c) Dynamical exponents obtained via LSWT for a larger system, $N=512$.

central spin and $B$ denotes the complementary subsystem; see inset in Fig. 4(a).

Figure 4(a) shows a typical TDVP result for the von Neumann entropy, $n \rightarrow 1$ [71], and the same local quench as in Fig. 3(a). Similar results are found for the other Rényi entropies [70]. We find that $\mathcal{S}_{n}(R, t)$ is a monotonic function of both position and time, and no local maxima such as those found previously for the correlation function and the magnetization are observed. This is consistent with a causal spreading of information and the expectation that the entanglement entropy decreases with the size of the smaller partition $(A)$. The entanglement edge (EE) is clearly visible in Fig. 4(a) and a fit to the algebraic scaling law $t \sim R^{\beta_{\mathrm{EE}}^{n}}$ allows us to extract the exponent. Varying the threshold in a wide range, $\epsilon \in[0.2,0.8]$, we find $\beta_{\mathrm{EE}}^{n} \approx 1$ within error bars, irrespectively of the interaction range; see cyan points in Fig. 4(b). The error bars correspond to the variation of $\beta_{\mathrm{EE}}^{n}$ with $\epsilon$, which is due to finite-size effects (see below). Our results imply that the propagation of entanglement is close to ballistic in both the local and quasilocal regimes. This contrasts with the behavior of the one- and two-point observables considered thus far, which exhibit sub-ballistic causal edges. It is a direct consequence of the fact that the bipartite entanglement entropy is a highly nonlocal quantity, which takes into account all entangled pairs on either side of the bipartition $R$ [39,72-74].

More precisely, we have analytically computed the entanglement entropy within LSWT [70]. The results are in good agreement with those extracted via TDVP for the same system sizes; see Fig. 4(b). The ballistic spreading of entanglement is further confirmed by the LSWT results obtained in much larger systems, with significantly smaller error bars; see Fig. 4(c). Bipartitioning the quenched initial state within LSWT yields an entanglement Schmidt rank bounded by 2 . This is consistent with our TDVP results at all times and the saturation of the entanglement entropies to $\mathcal{S}_{n}(R, t \rightarrow$ $\infty) \simeq \log (2) \simeq 0.69$; see Fig. 4(c). The two eigenvalues of the reduced density matrix $\hat{\rho}_{A}, \lambda_{1}(R, t)$, and $\lambda_{2}(R, t)=1-$ $\lambda_{1}(R, t)$, can then be computed analytically. In the asymptotic limit and for not too small values of $R / t$, we find $\lambda_{2}(R, t) \propto t^{\frac{1}{2-\alpha}} \zeta\left(\frac{3-\alpha}{2-\alpha}, R\right) \sim(t / R)^{\frac{1}{2-\alpha}}$, with $\zeta$ the Hurwitz zeta function [75,76]. Hence, the $n$-order Rényi entropy is a function of the ratio $R / t$,

$$
\mathcal{S}_{n}(R, t) \simeq \frac{1}{1-n} \log \left\{\lambda_{1}(R / t)^{n}+\lambda_{2}(R / t)^{n}\right\} .
$$

This confirms the ballistic propagation of the entanglement entropy $\left(\beta_{\mathrm{EE}}^{n}=1\right)$ consistent with the results of Fig. 4 for $n=$ 1 and other Rényi orders $n$ [70].

Conclusion. Our results show the emergence of a weak form of causality in the intermediate regime of the long-range Ising model, characterized by algebraic propagation laws with exponents that depend on the observables and the range of interactions. While local spins and spin correlations both have a sub-ballistic propagation edge, $t \propto R^{\beta}$, with $\beta>1$, the causal region is characterized by local maxima propagating super-ballistically and ballistically, respectively. The distinction between the causal edge and the local maxima, which can show drastically different dynamical behaviors, is thus pivotal in the characterization of causality in long-range quantum systems. In contrast, the propagation of entanglement is ballistic in both the local and quasilocal regimes, and the causal region is featureless.

These results call for future experimental and theoretical work. On the one hand, our predictions are directly relevant to quantum simulators using, for instance, trapped ions, where the interaction range can be controlled. While analysis of spin and correlation spreading in first experiments have been limited by finite-size effects [64,65], systems with more than 50 ions, comparable to the system size used in our simulations, are now accessible [77] and Rényi entanglement entropies can now be measured in trapped ion platforms [78,79] and digital quantum computers [80]. On the other hand, it would be interesting to further test the robustness of the observed algebraic scaling laws by quantitatively investigating the dependence (if any) of the exponents on the strength of the quenches, the phases of the model, and their values in different models, such as the long-range XY $[9,63]$, Heisenberg $[81,82]$, and Hubbard [44,58] models, as well as in dimensions higher than one. This extended analysis could allow the identification of dynamical universality classes, i.e., models which share the same algebraic laws for correlations out of equilibrium.

Acknowledgments. We thank the CPHT computer team for valuable support. The numerical calculations were performed using HPC resources from GENCI-CINES (Grant No. 2019-A0070510300). We acknowledge use of the ITENSORS.JL software package [83]. L.T. acknowledges support from the Ramón y Cajal (Program No. RYC-2016-20594), the "Plan Nacional Generación de Conocimiento" Grant No. PGC2018095862-B-C22, and the State Agency for Research of the Spanish Ministry of Science and Innovation through the "Unit of Excellence María de Maeztu 2020-2023" award to the Institute of Cosmos Sciences (Grant No. CEX2019-000918-M). J.T.S. and J.D. contributed equally to this work. 
[1] T. Dauxois, S. Ruffo, E. Arimondo, and M. Wilkens (eds.), Dynamics and Thermodynamics of Systems with Long Range Interactions, Lecture Notes in Physics (Springer-Verlag, Berlin/Heidelberg, 2002).

[2] M. Kastner, Nonequivalence of Ensembles for Long-Range Quantum Spin Systems in Optical Lattices, Phys. Rev. Lett. 104, 240403 (2010); Nonequivalence of ensembles in the CurieWeiss anisotropic quantum Heisenberg model, J. Stat. Mech. (2010) P07006.

[3] N. D. Mermin and H. Wagner, Absence of Ferromagnetism or Antiferromagnetism in One- Or Two-Dimensional Isotropic Heisenberg Models, Phys. Rev. Lett. 17, 1133 (1966).

[4] P. C. Hohenberg, Existence of long-range order in one and two dimensions, Phys. Rev. 158, 383 (1967).

[5] D. Peter, S. Müller, S. Wessel, and H. P. Büchler, Anomalous Behavior of Spin Systems with Dipolar Interactions, Phys. Rev. Lett. 109, 025303 (2012).

[6] J. Eisert, M. Cramer, and M. B. Plenio, Colloquium: Area laws for the entanglement entropy, Rev. Mod. Phys. 82, 277 (2010).

[7] T. Koffel, M. Lewenstein, and L. Tagliacozzo, Entanglement Entropy for the Long-Range Ising Chain in a Transverse Field, Phys. Rev. Lett. 109, 267203 (2012).

[8] A. Cadarso, M. Sanz, M. M. Wolf, J. I. Cirac, and D. PérezGarcía, Entanglement, fractional magnetization, and long-range interactions, Phys. Rev. B 87, 035114 (2013).

[9] J. Schachenmayer, B. P. Lanyon, C. F. Roos, and A. J. Daley, Entanglement Growth in Quench Dynamics with Variable Range Interactions, Phys. Rev. X 3, 031015 (2013).

[10] W. Thirring, Systems with negative specific heat, Z. Phys. 235, 339 (1970).

[11] I. Buluta and F. Nori, Quantum simulators, Science 326, 108 (2009)

[12] I. M. Georgescu, S. Ashhab, and F. Nori, Quantum simulation, Rev. Mod. Phys. 86, 153 (2014).

[13] J. I. Cirac and P. Zoller, Goals and opportunities in quantum simulation, Nat. Phys. 8, 264 (2012); I. Bloch, J. Dalibard, and S. Nascimbène, Quantum simulations with ultracold quantum gases, ibid. 8, 267 (2012); R. Blatt and C. F. Roos, Quantum simulations with trapped ions, ibid. 8, 277 (2012); A. AspuruGuzik and P. Walther, Photonic quantum simulators, ibid. 8, 285 (2012); A. A. Houck, H. E. Tureci, and J. Koch, On-chip quantum simulation with superconducting circuits, ibid. 8, 292 (2012).

[14] C. Gross and I. Bloch, Quantum simulations with ultracold atoms in optical lattices, Science 357, 995 (2017).

[15] L. Sanchez-Palencia, Quantum simulation: From basic principles to applications, C. R. Phys. 19, 357 (2018); L. Tarruell and L. Sanchez-Palencia, Quantum simulation of the Hubbard model with ultracold fermions in optical lattices, ibid. 19, 365 (2018); M. Aidelsburger, S. Nascimbene, and N. Goldman, Artificial gauge fields in materials and engineered systems, ibid. 19, 394 (2018); J. Lebreuilly and I. Carusotto, Quantum simulation of zero temperature quantum phases and incompressible states of light via non-Markovian reservoir engineering techniques, ibid. 19, 433 (2018); K. Le Hur, L. Henriet, L. Herviou, K. Plekhanov, A. Petrescu, T. Goren, M. Schiro, C. Mora, and P. P. Orth, Driven dissipative dynamics and topology of quantum impurity systems, ibid. 19, 451 (2018); M. Bell, B. Douçot, M. Gershenson, L. Ioffe, and A. Petkovic, Josephson ladders as a model system for 1d quantum phase transitions, ibid. 19, 484 (2018); F. Alet and N. Laflorencie, Many-body localization: An introduction and selected topics, ibid. 19, 498 (2018).

[16] X.-L. Deng, D. Porras, and J. I. Cirac, Effective spin quantum phases in systems of trapped ions, Phys. Rev. A 72, 063407 (2005).

[17] R. Islam, E. Edwards, K. Kim, S. Korenblit, C. Noh, H. Carmichael, G.-D. Lin, L.-M. Duan, C.-C. Joseph Wang, J. Freericks et al., Onset of a quantum phase transition with a trapped ion quantum simulator, Nat. Commun. 2, 377 (2011).

[18] B. P. Lanyon, C. Hempel, D. Nigg, M. Müller, R. Gerritsma, F. Zähringer, P. Schindler, J. T. Barreiro, M. Rambach, G. Kirchmair et al., Universal digital quantum simulation with trapped ions, Science 334, 57 (2011).

[19] C. Schneider, D. Porras, and T. Schaetz, Experimental quantum simulations of many-body physics with trapped ions, Rep. Prog. Phys. 75, 024401 (2012).

[20] A. Bermudez, L. Tagliacozzo, G. Sierra, and P. Richerme, Long-range Heisenberg models in quasiperiodically driven crystals of trapped ions, Phys. Rev. B 95, 024431 (2017).

[21] V. Bendkowsky, B. Butscher, J. Nipper, J. P. Shaffer, R. Löw, and T. Pfau, Observation of ultralong-range Rydberg molecules, Nature (London) 458, 1005 (2009).

[22] H. Weimer, M. Müller, I. Lesanovsky, P. Zoller, and H. P. Büchler, A Rydberg quantum simulator, Nat. Phys. 6, 382 (2010).

[23] P. Schausz, M. Cheneau, M. Endres, T. Fukuhara, S. Hild, A. Omran, T. Pohl, C. Gross, S. Kuhr, and I. Bloch, Observation of spatially ordered structures in a two-dimensional Rydberg gas, Nature (London) 491, 87 (2012).

[24] A. Browaeys, D. Barredo, and T. Lahaye, Experimental investigations of dipole-dipole interactions between a few Rydberg atoms, J. Phys. B: At. Mol. Opt. Phys. 49, 152001 (2016).

[25] A. Griesmaier, J. Werner, S. Hensler, J. Stuhler, and T. Pfau, Bose-Einstein Condensation of Chromium, Phys. Rev. Lett. 94, 160401 (2005).

[26] Q. Beaufils, R. Chicireanu, T. Zanon, B. Laburthe-Tolra, E. Maréchal, L. Vernac, J.-C. Keller, and O. Gorceix, All-optical production of chromium Bose-Einstein condensates, Phys. Rev. A 77, 061601 (2008).

[27] M. Lu, N. Q. Burdick, S. H. Youn, and B. L. Lev, Strongly Dipolar Bose-Einstein Condensate of Dysprosium, Phys. Rev. Lett. 107, 190401 (2011).

[28] S. Baier, M. J. Mark, D. Petter, K. Aikawa, L. Chomaz, Z Cai, M. Baranov, P. Zoller, and F. Ferlaino, Extended BoseHubbard models with ultracold magnetic atoms, Science 352, 201 (2016).

[29] T. Lahaye, C. Menotti, L. Santos, M. Lewenstein, and T. Pfau, The physics of dipolar bosonic quantum gases, Rep. Prog. Phys. 72, 126401 (2009).

[30] A. Micheli, G. K. Brennen, and P. Zoller, A toolbox for latticespin models with polar molecules, Nat. Phys. 2, 341 (2006).

[31] B. Yan, S. A. Moses, B. Gadway, J. P. Covey, K. R. A. Hazzard, A. M. Rey, D. S. Jin, and J. Ye, Observation of dipolar spinexchange interactions with lattice-confined polar molecules, Nature (London) 501, 521 (2013).

[32] S. A. Moses, J. P. Covey, M. T. Miecnikowski, D. S. Jin, and J. Ye, New frontiers for quantum gases of polar molecules, Nat. Phys. 13, 13 (2017). 
[33] O. Firstenberg, T. Peyronel, Q.-Y. Liang, A. V. Gorshkov, M. D. Lukin, and V. Vuletic, Attractive photons in a quantum nonlinear medium, Nat. Phys. 502, 71 (2013).

[34] L. Childress, M. V. Gurudev Dutt, J. M. Taylor, A. S. Zibrov, F. Jelezko, J. Wrachtrup, P. R. Hemmer, and M. D. Lukin, Coherent dynamics of coupled electron and nuclear spin qubits in diamond, Science 314, 281 (2006).

[35] G. Balasubramanian, P. Neumann, D. Twitchen, M. Markham, R. Kolesov, N. Mizuochi, J. Isoya, J. Achard, J. Beck, J. Tissler et al., Ultralong spin coherence time in isotopically engineered diamond, Nat. Mater. 8, 383 (2009).

[36] F. Dolde, I. Jakobi, B. Naydenov, N. Zhao, S. Pezzagna, C. Trautmann, J. Meijer, P. Neumann, F. Jelezko, and J. Wrachtrup, Room-temperature entanglement between single defect spins in diamond, Nat. Phys. 9, 139 (2013).

[37] E. H. Lieb and D. W. Robinson, The finite group velocity of quantum spin systems, Commun. Math. Phys. 28, 251 (1972).

[38] M. B. Hastings and T. Koma, Spectral gap and exponential decay of correlations, Commun. Math. Phys. 265, 781 (2006).

[39] P. Calabrese and J. Cardy, Time Dependence of Correlation Functions Following a Quantum Quench, Phys. Rev. Lett. 96, 136801 (2006).

[40] M. Cheneau, P. Barmettler, D. Poletti, M. Endres, P. Schauss, T. Fukuhara, C. Gross, I. Bloch, C. Kollath, and S. Kuhr, Lightcone-like spreading of correlations in a quantum many-body system, Nature (London) 481, 484 (2012).

[41] T. Fukuhara, P. Schausz, M. Endres, S. Hild, M. Cheneau, I. Bloch, and C. Gross, Microscopic observation of magnon bound states and their dynamics, Nature (London) 502, 76 (2013).

[42] R. Geiger, T. Langen, I. E. Mazets, and J. Schmiedmayer, Local relaxation and light-cone-like propagation of correlations in a trapped one-dimensional Bose gas, New J. Phys. 16, 053034 (2014).

[43] A. M. Läuchli and C. Kollath, Spreading of correlations and entanglement after a quench in the one-dimensional BoseHubbard model, J. Stat. Mech. (2008) P05018.

[44] S. R. Manmana, S. Wessel, R. M. Noack, and A. Muramatsu, Time evolution of correlations in strongly interacting fermions after a quantum quench, Phys. Rev. B 79, 155104 (2009).

[45] P. Barmettler, D. Poletti, M. Cheneau, and C. Kollath, Propagation front of correlations in an interacting Bose gas, Phys. Rev. A 85, 053625 (2012).

[46] G. Carleo, F. Becca, L. Sanchez-Palencia, S. Sorella, and M. Fabrizio, Light-cone effect and supersonic correlations in oneand two-dimensional bosonic superfluids, Phys. Rev. A 89, 031602(R) (2014).

[47] J. Despres, L. Villa, and L. Sanchez-Palencia, Twofold correlation spreading in a strongly correlated lattice Bose gas, Sci. Rep. 9, 4135 (2019).

[48] L. Villa, J. Despres, and L. Sanchez-Palencia, Unraveling the excitation spectrum of many-body systems from quantum quenches, Phys. Rev. A 100, 063632 (Dec 2019).

[49] L. Villa, J. Despres, S. J. Thomson, and L. Sanchez-Palencia, Local quench spectroscopy of many-body quantum systems, Phys. Rev. A 102, 033337 (2020).

[50] P. Hauke and L. Tagliacozzo, Spread of Correlations in LongRange Interacting Quantum Systems, Phys. Rev. Lett. 111, 207202 (2013).
[51] J. Eisert, M. van den Worm, S. R. Manmana, and M. Kastner, Breakdown of Quasilocality in Long-Range Quantum Lattice Models, Phys. Rev. Lett. 111, 260401 (2013).

[52] M. Foss-Feig, Z.-X. Gong, C. W. Clark, and A. V. Gorshkov, Nearly Linear Light Cones in Long-Range Interacting Quantum Systems, Phys. Rev. Lett. 114, 157201 (2015).

[53] D. V. Else, F. Machado, C. Nayak, and N. Y. Yao, Improved Lieb-Robinson bound for many-body Hamiltonians with power-law interactions, Phys. Rev. A 101, 022333 (2020).

[54] L. Cevolani, G. Carleo, and L. Sanchez-Palencia, Spreading of correlations in exactly solvable quantum models with longrange interactions in arbitrary dimensions, New J. Phys. 18, 093002 (2016).

[55] C.-F. Chen and A. Lucas, Finite Speed of Quantum Scrambling with Long Range Interactions, Phys. Rev. Lett. 123, 250605 (2019).

[56] M. C. Tran, C.-F. Chen, A. Ehrenberg, A. Y. Guo, A. Deshpande, Y. Hong, Z.-X. Gong, A. V. Gorshkov, and A. Lucas, Hierarchy of Linear Light Cones with Long-Range Interactions, Phys. Rev. X 10, 031009 (2020).

[57] T. Kuwahara and K. Saito, Strictly Linear Light Cones in LongRange Interacting Systems of Arbitrary Dimensions, Phys. Rev. X 10, 031010 (2020).

[58] L. Cevolani, G. Carleo, and L. Sanchez-Palencia, Protected quasi-locality in quantum systems with long-range interactions, Phys. Rev. A 92, 041603(R) (2015).

[59] J. Schachenmayer, A. Pikovski, and A. M. Rey, Dynamics of correlations in two-dimensional quantum spin models with long-range interactions: A phase-space Monte-Carlo study, New J. Phys. 17, 065009 (2015).

[60] A. S. Buyskikh, M. Fagotti, J. Schachenmayer, F. Essler, and A. J. Daley, Entanglement growth and correlation spreading with variable-range interactions in spin and fermionic tunneling models, Phys. Rev. A 93, 053620 (2016).

[61] D. J. Luitz and Y. Bar Lev, Emergent locality in systems with power-law interactions, Phys. Rev. A 99, 010105(R) (2019).

[62] L. Colmenarez and D. J. Luitz, Lieb-Robinson bounds and outof-time order correlators in a long-range spin chain, Phys. Rev. Research 2, 043047 (2020).

[63] L. Cevolani, J. Despres, G. Carleo, L. Tagliacozzo, and L. Sanchez-Palencia, Universal scaling laws for correlation spreading in quantum systems with short- and long-range interactions, Phys. Rev. B 98, 024302 (2018).

[64] P. Richerme, Z.-X. Gong, A. Lee, C. Senko, J. Smith, M. FossFeig, S. Michalakis, A. V. Gorshkov, and C. Monroe, Non-local propagation of correlations in quantum systems with long-range interactions, Nature (London) 511, 198 (2014).

[65] P. Jurcevic, B. P. Lanyon, P. Hauke, C. Hempel, P. Zoller, R. Blatt, and C. F. Roos, Quasiparticle engineering and entanglement propagation in a quantum many-body system, Nature (London) 511, 202 (2014).

[66] D. Jaksch, J. I. Cirac, P. Zoller, S. L. Rolston, R. Côté, and M. D. Lukin, Fast Quantum Gates for Neutral Atoms, Phys. Rev. Lett. 85, 2208 (2000).

[67] M. D. Lukin, M. Fleischhauer, R. Cote, L. M. Duan, D. Jaksch, J. I. Cirac, and P. Zoller, Dipole Blockade and Quantum Information Processing in Mesoscopic Atomic Ensembles, Phys. Rev. Lett. 87, 037901 (2001).

[68] M. F. Maghrebi, Z.-X. Gong, M. Foss-Feig, and A. V. Gorshkov, Causality and quantum criticality in 
long-range lattice models, Phys. Rev. B 93, 125128 (2016).

[69] J. Haegeman, B. Pirvu, D. J. Weir, J. I. Cirac, T. J. Osborne, H. Verschelde, and F. Verstraete, Variational matrix product ansatz for dispersion relations, Phys. Rev. B 85, 100408 (2012).

[70] See Supplemental Material at http://link.aps.org/supplemental/ 10.1103/PhysRevResearch.3.L012022 for details on the $\epsilon$ method for determining the correlation, spin, and entanglement edges, the spreading of spin correlations along the $x$ direction as well as strong quenches, and the LSWT for the spreading of the local magnetization and entanglement entropies; see also Refs. $[84,85]$.

[71] The von Neumann entropy also reads as $\mathcal{S}_{\mathrm{vN}}=\mathcal{S}_{n \rightarrow 1}(R, t)=$ $-\operatorname{Tr}\{\hat{\rho}(R, t) \log [\hat{\rho}(R, t)]\}$.

[72] V. Alba and P. Calabrese, Entanglement and thermodynamics after a quantum quench in integrable systems, Proc. Natl. Acad. Sci. USA 114, 7947 (2017).

[73] A. Bastianello and P. Calabrese, Spreading of entanglement and correlations after a quench with intertwined quasiparticles, SciPost Phys. 5, 033 (2018).

[74] P. Calabrese, Entanglement spreading in non-equilibrium integrable systems, SciPost Phys. Lect. Notes 20 (2020).

[75] A. Hurwitz, in Einige Eigenschaften der Dirichletschen Functionen $F(s)=\sum\left(\frac{D}{n}\right) \cdot \frac{1}{n}$, die bei der Bestimmung der Klassenanzahlen binärer quadratischer Formen auftreten (Springer Basel, Basel, 1932), pp. 72-88.

[76] W. Magnus, F. Oberhettinger, and R. P. Soni, The gamma function and related functions, in Formulas and Theorems for the Special Functions of Mathematical Physics (Springer, Berlin, Heidelberg, 1966), pp. 1-37.
[77] J. Zhang, G. Pagano, P. W. Hess, A. Kyprianidis, P. Becker, H. Kaplan, A. V. Gorshkov, Z.-X. Gong, and C. Monroe, Observation of a many-body dynamical phase transition with a 53-qubit quantum simulator, Nature (London) 551, 601 (2017).

[78] N. M. Linke, S. Johri, C. Figgatt, K. A. Landsman, A. Y. Matsuura, and C. Monroe, Measuring the Rényi entropy of a two-site Fermi-Hubbard model on a trapped ion quantum computer, Phys. Rev. A 98, 052334 (2018).

[79] T. Brydges, A. Elben, P. Jurcevic, B. Vermersch, C. Maier, B. P. Lanyon, P. Zoller, R. Blatt, and C. F. Roos, Probing Rényi entanglement entropy via randomized measurements, Science 364, 260 (2019).

[80] J. Vovrosh and J. Knolle, Confinement and entanglement dynamics on a digital quantum computer, arXiv:2001.03044.

[81] L. Yang and A. E. Feiguin, From deconfined spinons to coherent magnons in an antiferromagnetic Heisenberg chain with long range interactions, arXiv:2005.09086.

[82] L. Yang, P. Weinberg, and A. E. Feiguin, Quantum criticality in antiferromagnetic quantum spin ladders with long-range interactions, arXiv:2012.14908.

[83] M. Fishman, S. R. White, and E. M. Stoudenmire, The ITensor software library for tensor network calculations, arXiv:2007.14822.

[84] T. Holstein and H. Primakoff, Field dependence of the intrinsic domain magnetization of a ferromagnet, Phys. Rev. 58, 1098 (1940).

[85] N. Bogoliubov, On the theory of superfluidity, J. Phys. 11, 23 (1947). 